\title{
Les proches des personnes atteintes de démence disent oui à la technologie
}

Natascha Radenovic

L'utilisation de la technique pour les personnes atteintes de démence fait partie des questions d'éthique les plus controversées en matière de prise en charge des personnes concernées. Dans le cadre d'un travail de Master, des entretiens avec les proches des personnes concernées ont montré que la technologie ne remplacerait jamais la proximité humaine, mais qu'elle pouvait être une distraction agréable dans le quotidien des personnes atteintes de démence avec une grande importance accordée à l'adaptation individuelle surtout.

\section{L'utilisation de la technique suscite l'intérêt des médias}

Depuis que le Centre de compétences en démence (Domicil Kompetenzzentrum Demenz) Bethlehemacker dispose de différentes offres technologiques, il est régulièrement présent dans les médias. Les zones de résidence de l'institution de soins de longue durée de Berne ont été adaptées et réaménagées en 2013 en fonction des besoins des personnes atteintes de démence. Le but ultime de cette planification et de la réalisation des transformations était d'offrir aux personnes atteintes de démence une qualité de vie élevée et des expériences émotionnelles positives. Avec ce projet innovant, tous les participants entraient inévitablement en terrain inconnu. Ainsi pas de technologie de sécurité et d'orientation placée au centre, mais plutôt des offres technologiques que les résidents atteints de démence peuvent utiliser, comme par exemple cheminée/aquarium sous forme de film à la télévision, douche de lumière, siège massant, lit à eau avec lumière, boule à facette, « cascade à toucher » (ou « spaghetti » comme certains résidents l'appellent), jacuzzi, «colonnes lumineuses » et un compartiment de train.

\section{Questions d'éthique}

Les discussions concernant l'application et l'utilisation de cette technologie posent surtout des questions d'éthique : la réalité doit-elle être simulée aux personnes atteintes de démence ? Quels conflits éthiques résultent de l'utilisation de la technologie ? La technologie remplace-t-elle la proximité humaine ?

Le Kompetenzzentrum Demenz Bethlehemacker a effectué une évaluation des effets de la technologie avec l'Institut de l'âge de la Haute école spécialisée bernoise (BHF). L'un des groupes cibles de l'évaluation était les proches des personnes atteintes de démence. Dans le cadre d'un travail de Master 
à l'Institut de l'âge de la BHF, 12 proches ont été interrogés sur leur perception et leur évaluation de l'utilisation de la technologie dans trois enquêtes distantes de six semaines chacune.

\section{Entretien avec les proches des personnes atteintes de démence}

L'exploitation des entretiens a montré que les proches étaient ouverts à la technologie et qu'ils pensaient qu'elle était positive. Selon eux, elle apporte du changement dans le quotidien des personnes atteintes de démence, mais également dans celle des visiteurs. Les proches ont déclaré que l'ambiance était agréable et sympathique. Ils ont observé que les résidents utilisaient la technologie de préférence avec d'autres personnes. Les résidents peuvent eux-mêmes manier et utiliser quelques-unes des applications technologiques, comme par exemple le jacuzzi. Par conséquent, ils sont toujours accompagnés des proches ou des soignants. L'utilisation de la technologie adaptée aux préférences et aux souhaits individuels des personnes atteintes de démence est importante pour les proches. Ils ont également rapporté que les résidents délaissaient eux-mêmes la technologie quand ils en avaient assez et quand le contenu du film ne leur correspondait plus.

Les proches ont également fait part de nombreuses bonnes idées pour l'utilisation et l'optimisation de la technologie et son application. Ainsi permettre par exemple une manipulation simple, avec le bouton Marche/Arrêt par exemple, ou un accès par code qui permettrait aux proches d'utiliser la technologie de manière autonome lors des visites. En plus des souhaits des proches, les entretiens ont également fait ressortir des recommandations concernant la technologie qui ont été utilisées en tant que précieuses indications dans le centre de compétences Domicil Kompetenzzentrum Demenz Bethlehemacker. Ainsi, il a par exemple été proposé :

- d'encourager l'individualité des résidents avec une offre diversifiée de films,

- de simplifier au maximum l'utilisation (p. ex. marche/arrêt) des technologies proposées afin que les proches puissent également les utiliser de manière autonome,

- de mieux initier les proches à l'utilisation (autonome) des offres et

- d'institutionnaliser les échanges entre les proches et les soignants relatifs à la technologie.

\section{Conclusion}

Bien que l'utilisation de la technologie dans la prise en charge de personnes atteintes de démence comporte de nombreuses questions d'éthique, les proches n'ont signalé aucun conflit éthique, ni au sein de l'institution ni en dehors par exemple dans les conversations avec les connaissances ou d'autres personnes. Comme les personnes atteintes de démence dépendent en majorité de l'aide humaine, la technologie ne peut en aucun cas remplacer l'attention humaine, mais constituer une aide bienvenue et agréable et un changement dans le quotidien. 


\section{Littérature :}

Radenovic, N. (2015)Technik und ihre Auswirkungen auf die Menschen mit Demenz aus der Sicht der Angehörigen. MAS „Gerontologie- Altern: Lebensgestaltung 50+“. Institut Alter, Berner Fachhochschule (BFH).

Une littérature plus complète peut être obtenue auprès de l'auteure.

Auteure : Natascha Radenovic est infirmière spécialiste et directrice de la prise en charge et des soins au Domicil Steigerhubel de Berne.

Contact : natascha.radenovic@domicilbern.ch 